\title{
Duration and Risk Factors of Post-COVID Symptoms Following Recovery Among the Medical Doctors in Bangladesh
}

Sarmin Sultana ${ }^{1}$, Mohammad Tanvir Islam ${ }^{2}$, Marium Salwa ${ }^{1}$, Shah M. Zakir Hossain ${ }^{3}$, Md Nazmul Hasan ${ }^{2}$, Abdullah A. Masum ${ }^{2}$, Abed H. Khan ${ }^{2}$, Md Maruf Haque Khan ${ }^{1}$, M Atiqul Haque ${ }^{1}$

1. Department of Public Health and Informatics, Bangabandhu Sheikh Mujib Medical University, Dhaka, BGD 2. Department of Internal Medicine, Bangabandhu Sheikh Mujib Medical University, Dhaka, BGD 3. Department of Nephrology, Bangabandhu Sheikh Mujib Medical University, Dhaka, BGD

Corresponding author: Sarmin Sultana, sarmindoc@gmail.com

\section{Abstract}

A large number of coronavirus disease 2019 (COVID-19) recovered patients are suffering from related symptoms. We conducted telephone interviews with 186 COVID-19 recovered medical doctors to determine the post-COVID symptoms, duration, and associated risk factors. About $70 \%$ of participants had at least one acute post-COVID symptom, including fatigue (43.0\%), sleep disturbance (13.4\%), lack of concentration (11.8\%), breathing difficulty (10.2\%), headache (6.5\%), and muscle pain (6.5\%). However, about $24 \%$ of participants reported having long post-COVID symptoms. Logistic regression analysis showed that female sex (odds ratio $\{\mathrm{OR}\}, 2.79 ; 95 \% \mathrm{CI}, 1.28-6.06$; p-value: 0.010$)$ and comorbid conditions (OR, $2.28 ; 95 \% \mathrm{CI}$, 1.08-4.79; p: value, 0.030) are risk factors for the long post-COVID symptoms.

Categories: Internal Medicine, Infectious Disease, Epidemiology/Public Health

Keywords: covid-19, medical doctors, duration, risk factors, post-covid symptoms

\section{Introduction}

Around 131 million people have been infected worldwide since the outbreak of coronavirus disease 2019 (COVID-19) in December 2019, while approximately 2.9 million people died and 106 million people reportedly recovered as of April 4, 2021 [1]. The initial focus of the healthcare providers, epidemiologists, and policymakers was to identify the epidemiological and clinical features of COVID-19, its management, and prevention strategies as an emerging infectious disease. Medical professionals and researchers consequently concentrated on estimating the disease burden of COVID-19 among the survivors.

Since recovering from COVID-19, several patients complained of persistent or new symptoms [2,3]. Cough, shortness of breath, nausea, headache, palpitations, chest pain, joint pain, physical limitations, vision changes, hearing loss, loss of taste or smell, reduced mobility, numbness in extremities, tremors, myalgia, memory loss, cognitive impairment, mood changes, and others are some of the symptoms reported $[4,5]$.

Review began 05/08/2021 Review ended 05/26/2021 Published 05/31/2021

○ Copyright 2021

Sultana et al. This is an open access article distributed under the terms of the Creative Commons Attribution License CC-BY 4.0., which permits unrestricted use, distribution, and reproduction in any medium, provided the original author and source are credited.
The terms "long COVID," "post-COVID syndrome," "long haulers," "post-COVID symptoms," and "post-acute COVID-19 syndrome" have been used in the absence of a consensus about how to define these conditions $[6]$.

Several factors have been reported as associated factors for post-COVID symptoms, including increasing age, female sex, presence of comorbidities, psychiatric disorders, obesity, hospital admission, early symptoms, irregular findings in auscultation at symptom onset, and so on $[3,7,8]$. Younger patients, including those with mild symptoms during the acute phase of infection, are presenting with similar complaints $[2,4,9]$.

Post-COVID symptoms should be identified by health professionals; otherwise, some of the essential features or risk factors for post-COVID symptoms can be ignored, which may carry severe consequences [4]. The current research aimed to estimate the prevalence, length of illness, and risk factors of post-COVID symptoms in order to warn healthcare professionals about the imminent burden of the disease and recognize people at higher risk of suffering.

\section{Materials And Methods}

Medical doctors are one of the most vulnerable groups for getting COVID-19 infection. Our study was designed to estimate the prevalence of acute post-COVID symptoms and long post-COVID symptoms among the recovered medical doctors, and find out the risk factors of long post-COVID symptoms.

\section{Study design}

This descriptive cross-sectional analysis was carried out among Bangladeshi medical doctors who had been infected with COVID-19 from April 1, 2020, to July 30, 2020. The interval between the participants' COVID- 
19 detection by a positive reverse transcription-polymerase chain reaction (RT-PCR) for severe acute respiratory syndrome coronavirus 2 (SARS-CoV-2) and the date of data collection was 124.4 (21.6) days on average. The study participants were chosen with the assumption that medical doctors are more knowledgeable of the clinical features of COVID-19 and are more likely to share relevant details than the general public.

Participants in the sample were those who had recovered from acute COVID-19 infection at least two months prior to the date of data collection. Recovery from acute COVID-19 infection was considered when the patient had no fever for three consecutive days without medication or improved in COVID-19 related symptoms or was discharged in case of hospitalization or had two subsequent negative RT-PCR results for SARS-CoV-2, 24 hours apart. The post-acute phase was described as the time period following recovery from acute infection. The post-acute phase was defined as at least two weeks after diagnosis for asymptomatic patients.

\section{Study population and sampling}

The Bangladesh Doctors' Foundation, an online philanthropic social network of medical doctors, provided a list of 540 COVID-19 RT-PCR test positive medical doctors with email addresses and mobile phone numbers. Using the formula given by Lwanga and Lemeshow, we estimated the sample size to be 192 based on the unknown prevalence of post-COVID-19 symptoms among all infected patients and a 7\% margin of error [10]. After accounting for a 30\% non-response rate (from the author's previous survey of medical doctors during the COVID-19 pandemic), our final sample size was 250. Using the Statistical Package for Social Sciences (SPSS) program, samples were drawn randomly from the list of COVID-19 positive medical doctors. The doctors who were unwilling to participate or could not be reached over the phone even after trying for three consecutive days were excluded from the study.

\section{Data collection}

The data were collected through telephone interviews over three weeks. For data collection, five resident medical doctors from the Department of Internal Medicine at Bangabandhu Sheikh Mujib Medical University were recruited. A five-day training session was arranged, during which data collectors were taught how to present themselves, explain the purpose of the research, gain informed consent, and collect data using mobile phones. Data collectors scheduled each participant's time in advance, and each interview took about 15 minutes to complete. After receiving approval from the participants, the interviews were audiotaped.

A semi-structured questionnaire was developed to gather information on participants' clinico-demographic characteristics as well as post-COVID symptoms. Age, sex, comorbid conditions, smoking history, hospital admission, intensive care unit (ICU) support, and complication during acute COVID-19 infection were included in the clinico-demographic profile. The smoking history was categorized as never smoker, past smoker (who quit smoking six months ago), and current smoker. Past smokers and never smokers were considered non-smoker during analysis.

The self-reported data on comorbidity were obtained regarding non-communicable diseases (NCDs) like hypertension, diabetes, asthma, thyroid disease, ischemic heart disease, chronic kidney diseases, and chronic liver disease. The participants who reported laboratory-confirmed bacterial coinfection, acute respiratory distress syndrome (ARDS), acute cardiac injury, acute kidney injury, multiorgan dysfunction, sepsis, or shock during the acute phase of COVID-19 illness were considered as having associated complications.

The post-COVID symptoms were defined as the presence of signs and symptoms consistent with COVID-19 during the post-acute phase. Participants were asked whether they had post-COVID symptoms including trouble breathing, cough, chest pain, palpitation, nausea, sleep disruption, memory lapses, lack of concentration, anosmia, loss of taste, loss of appetite, weight loss, muscle pain, joint pain, hair fall, and rash. Participants were also asked how long they had experienced any of these symptoms. We classified the post-COVID symptoms based on their duration. Symptoms persisting $\leqslant 60$ days following recovery were considered as acute post-COVID symptoms and >60 days following recovery were considered as long postCOVID symptoms.

\section{Data analysis}

As summary measures for the categorical variables, frequencies and percentages were measured. Arithmetic means (standard deviation) were used to characterize the numerical results.

A logistic regression model was used to evaluate the relationship between any long post-COVID symptoms and explanatory variables. The variables with a p-value of 0.05 or less in the bivariate model or variables with a significant impact on outcome variables defined in empirical studies were chosen for further analysis in the logistic regression model to classify independent predictors of the outcome variable. The coefficient of inter-correlation with all of the other variables was found to be sufficient $(<0.235)$. The odds ratios (OR) were presented along with the corresponding 95\% confidence interval (CI). Data were analyzed using SPSS 


\section{Cureus}

version 21 (Armonk, NY: IBM Corp.) and the p-value was considered significant at 5\% level.

\section{Ethical consideration}

Ethical clearance was obtained from the Institutional Review Board of Bangabandhu Sheikh Mujib Medical University (BSMMU/2020/6317). Informed verbal consent was obtained from each study participant after explaining the study procedure, benefit and risk of participation, right to refuse to participate or withdraw from the study, confidential handling of data, and principal investigator's identity. The participants were at liberty of not responding to any question and withdraw from the research at any point.

\section{Results}

A total of 186 medical doctors were interviewed, with a $74.4 \%$ response rate.

\section{Clinico-demographic characteristics}

The participants' age ranged from 24 years to 75 years with a mean (SD) age of 34.8 (9.9) years. Approximately one-third of participants had at least one comorbid condition, with asthma (19.4\%) and hypertension (14.5\%) being the most commonly recorded. Approximately $20 \%$ of participants needed hospitalization, and $1.6 \%$ required ICU transfer. The incidence of at least one, two, and three or more postCOVID symptoms were $69.9 \%, 39.8 \%$, and $23.1 \%$, respectively (Table 1 ). 


\section{Cureus}

\begin{tabular}{|c|c|c|}
\hline Variables & Frequency & Percentage \\
\hline \multicolumn{3}{|l|}{ Age (in completed years) } \\
\hline$<50$ & 168 & 90.3 \\
\hline 50 to 59 & 10 & 5.4 \\
\hline$\geq 60$ & 8 & 4.3 \\
\hline \multicolumn{3}{|l|}{ Sex } \\
\hline Male & 123 & 66.1 \\
\hline Female & 63 & 33.9 \\
\hline \multicolumn{3}{|l|}{ Comorbidity } \\
\hline None & 117 & 62.9 \\
\hline Asthma & 36 & 19.4 \\
\hline Hypertension & 27 & 14.5 \\
\hline Diabetes & 14 & 7.5 \\
\hline Thyroid disease & 6 & 3.2 \\
\hline Ischemic heart disease & 2 & 1.1 \\
\hline \multicolumn{3}{|l|}{ Smoking status } \\
\hline Non-smoker ${ }^{\mathrm{a}}$ & 152 & 81.7 \\
\hline Current smoker & 25 & 13.4 \\
\hline Complication during acute phase & 13 & 7.0 \\
\hline Required hospital admission & 38 & 20.4 \\
\hline Required ICU support & 3 & 1.6 \\
\hline \multicolumn{3}{|l|}{ Post-COVID symptoms } \\
\hline At least one symptom & 130 & 69.9 \\
\hline At least two symptoms & 74 & 39.8 \\
\hline Three or more symptoms & 43 & 23.1 \\
\hline
\end{tabular}

TABLE 1: Clinico-demographic features of the participants ( $\mathrm{N}=186)$.

${ }^{a}$ Non-smoker $=$ Past smoker + Never smoker

ICU: intensive care unit; COVID: coronavirus disease

\section{Post-COVID symptoms and duration}

The participants reported that most of their post-COVID symptoms relieved within 30 days during the postacute phase. However, few symptoms like fatigue (8.1\%), difficulty in breathing (6.5\%), lack of concentration (4.8\%), hair fall (4.3\%), memory lapses (4.3\%), sleep disturbance (3.8\%), and joint pain (1.6\%) persisted $>60$ days even after recovery (Table 2).

Post-COVID symptoms

Duration during the post-acute phase

Frequency (percentage) ${ }^{\mathrm{a}}$ of participants

Total $\mathrm{N}=186$

$$
\text { Acute post-COVID symptoms }
$$

Long post-COVID symptoms

$<30$ days $\quad 31-60$ days $\quad>60$ days




\section{Cureus}

\begin{tabular}{|c|c|c|c|c|}
\hline \multicolumn{5}{|l|}{ Cardio-respiratory } \\
\hline Difficulty in breathing & $19(10.2)$ & $3(1.6)$ & $4(2.2)$ & $12(6.5)$ \\
\hline Cough & $12(6.5)$ & $10(5.4)$ & $2(1.1)$ & - \\
\hline Palpitation & $11(5.9)$ & $9(4.8)$ & $2(1.1)$ & - \\
\hline Chest pain & $5(2.7)$ & $3(1.6)$ & $1(0.5)$ & $1(0.5)$ \\
\hline Rhinorrhea & $2(1.1)$ & $2(1.1)$ & - & - \\
\hline Sore throat & $1(0.5)$ & $1(0.5)$ & - & - \\
\hline \multicolumn{5}{|l|}{ Neuro-psychiatric } \\
\hline Fatigue & $80(43.0)$ & $55(29.6)$ & $10(5.4)$ & $15(8.1)$ \\
\hline Sleep disturbance & $25(13.4)$ & $17(9.2)$ & $1(0.5)$ & $7(3.8)$ \\
\hline Lack of concentration & $22(11.8)$ & $10(5.4)$ & $3(1.6)$ & $9(4.8)$ \\
\hline Memory lapses & $13(7.0)$ & $4(2.2)$ & $1(0.5)$ & $8(4.3)$ \\
\hline Headache & $12(6.5)$ & $8(4.3)$ & $3(1.6)$ & $1(0.5)$ \\
\hline Anosmia & $11(5.9)$ & $7(3.8)$ & $4(2.2)$ & - \\
\hline Irritability & $8(4.3)$ & $6(3.2)$ & - & $2(1.1)$ \\
\hline Loss of taste & $6(3.2)$ & $4(2.2)$ & $1(0.5)$ & $1(0.5)$ \\
\hline Depressed mood & $3(2.6)$ & $3(2.6)$ & - & - \\
\hline Anxiety & $3(1.6)$ & $2(1.1)$ & - & $1(0.5)$ \\
\hline Vertigo & $2(1.1)$ & $2(1.1)$ & - & - \\
\hline \multicolumn{5}{|l|}{ Gastrointestinal } \\
\hline Loss of appetite & $7(3.8)$ & $6(3.2)$ & - & $1(0.5)$ \\
\hline Weight loss & $4(2.2)$ & $4(2.2)$ & - & - \\
\hline Diarrhea & $3(1.6)$ & $3(1.6)$ & - & - \\
\hline Abdominal pain & $1(0.5)$ & $1(0.5)$ & - & - \\
\hline Nausea & $1(0.5)$ & - & $1(0.5)$ & - \\
\hline \multicolumn{5}{|l|}{ Musculoskeletal } \\
\hline Muscle pain & $12(6.5)$ & $12(6.4)$ & - & - \\
\hline Joint pain & $8(4.3)$ & $5(2.7)$ & - & $3(1.6)$ \\
\hline \multicolumn{5}{|l|}{ Cutaneous } \\
\hline Hair fall & $9(4.8)$ & $1(0.5)$ & - & $8(4.3)$ \\
\hline Rash & $2(1.1)$ & $2(1.1)$ & - & - \\
\hline
\end{tabular}

\section{TABLE 2: Duration of post-COVID symptoms ( $\mathrm{N}=186)$.}

apercentage calculated for total participants.

COVID: coronavirus disease

\section{Risk factors of long post-COVID symptoms}

Among all the participants, 44 (23.7\%) reported suffering from at least one long post-COVID symptom. The adjusted logistic regression model for the persistence of any long post-COVID symptoms showed that female participants are 2.79 times more likely to suffer from long post-COVID symptoms than their male counterparts (OR, 2.79; 95\% CI, 1.28-6.06; p-value: 0.010). Participants with comorbid conditions are 2.28 


\section{Cureus}

times more likely to have persistent post-acute symptoms than others (OR, 2.28; 95\% CI, 1.08-4.79; p: value, 0.030) (Table 3).

\begin{tabular}{|c|c|c|}
\hline \multirow{2}{*}{ Variables } & \multicolumn{2}{|c|}{ Presence of long post-COVID symptom } \\
\hline & Unadjusted odds & Adjusted odds ${ }^{a}$ \\
\hline Age & $1.03(0.99-1.06)$ & $1.03(0.99-1.07)$ \\
\hline \multicolumn{3}{|l|}{ Sex } \\
\hline Male & Referent & Referent \\
\hline Female & $2.46(1.23-4.93)^{b}$ & $2.79(1.28-6.06)^{b}$ \\
\hline \multicolumn{3}{|l|}{ Comorbidity } \\
\hline Absent & Referent & Referent \\
\hline Present $^{d}$ & $2.58(1.29-5.16)^{\mathrm{C}}$ & $2.28(1.08-4.79)^{b}$ \\
\hline \multicolumn{3}{|l|}{ Smoking status } \\
\hline Non-smoker & Referent & Referent \\
\hline Current smoker & $0.39(0.11-1.40)$ & $0.62(0.16-2.34)$ \\
\hline \multicolumn{3}{|c|}{ Complication during acute phase } \\
\hline No & Referent & Referent \\
\hline Yes & $2.15(0.66-6.94)$ & $2.10(0.607-7.281)$ \\
\hline \multicolumn{3}{|c|}{ Hospital admission } \\
\hline No & Referent & Referent \\
\hline Yes & $1.19(0.53-2.71)$ & $1.04(0.42-2.59)$ \\
\hline
\end{tabular}

\section{TABLE 3: Risk factors for the long post-COVID symptom ( $\mathrm{N}=186)$.}

${ }^{\text {aAll }}$ of the risk factors listed in the table were simultaneously adjusted using logistic regression, with the persistence of at least one long postCOVID symptom.

${ }^{b} p$-Value $<0.05$

${ }^{c} p$-Value $<0.01$

dParticipant with any comorbid condition.

COVID: coronavirus disease

\section{Discussion}

Our study found that $70 \%$ of participants had post-COVID symptoms, where about $24 \%$ of the participants had at least one symptom for more than 60 days even after recovering from acute illness. Several recent studies reported diverse pictures of post-COVID symptoms with different durations. In a study from Italy, Carfi et al. found $87 \%$ of hospitalized COVID-19 patients had persisting symptoms related to COVID-19, where $43 \%$ had one or two symptoms, and $55 \%$ had three or more symptoms after an average of 60 days of their initial symptoms [11]. Similarly, Carvalho-Schneider et al. reported that $66 \%$ of their patients had long-COVID symptoms even after 60 days [8]. Tenforde et al. reported that about $35 \%$ of COVID-19 infected patients did not return to usual health after two to three weeks of their diagnosis in a multi-country study [7]. Apart from methodological variation, the absence of a uniform definition for the acute and post-acute phases makes the data incomparable across the world. Setting a post-COVID timeline will help determine whether the features are local or universal [12].

The findings of this study showed that the frequency of post-COVID symptoms decreased over time, as reported by other follow-up studies on COVID-19 [7,8]. This information is helpful for patient management. 
However, the duration and severity might vary based on the clinical and demographic characteristics of patients. These findings need further evaluation by close monitoring of the patients through follow-up studies. Several studies on other coronaviruses like severe acute respiratory syndrome (SARS) or Middle East respiratory syndrome (MERS) infection showed respiratory dysfunction, reduced exercise capacity, psychological problems such as post-traumatic stress disorder (PTSD), depression, and anxiety, and reduced quality of life beyond six months [13]. Wu et al. found that pulmonary fibrosis in CT findings persisted up to seven years post-infection with SARS [14]. Another study by Lam et al. revealed that $40 \%$ of people recovering from SARS had chronic fatigue symptoms 3.5 years after being diagnosed [15].

The most commonly reported post-COVID symptoms in the present study were fatigue, sleep disturbance, lack of concentration, breathing difficulty, memory lapse, myalgia, cough, etc. Carfì et al. found a high proportion of COVID-19 survivors suffered from fatigue (53\%) followed by dyspnea (43\%), joint pain (27\%), and chest pain (22\%) [11]. Anosmia/ageusia (59\%), dyspnea (30\%), or asthenia (40\%) were reported as common symptoms by Carvalho-Schneider et al. [8]. Garrigues et al. reported continued symptoms like fatigue (55\%), dyspnea (42\%), memory loss (34\%), sleep disorders (31\%), and difficulty with concentration (28\%) among recovered patients after three months of diagnosis [16]. With varied duration and frequency, these studies, however, reported an almost similar type of symptoms.

Our study findings suggest that females and participants with comorbid conditions are more likely to suffer from long post-COVID symptoms. Few other studies have also shown that females are at a higher risk of being a long hauler $[3,17]$. Tenforde et al. reported that having chronic medical conditions is a risk factor for persisting symptoms of COVID-19 [7].

There were some shortcomings in this report. Since this was a cross-sectional study rather than a follow-up study, there is a risk of recall bias. Furthermore, we could not determine whether post-COVID symptoms were chronic or newly formed acute phase symptoms. As the telephone interview method was used, the nonrespondents may also have varied from survey participants.

\section{Conclusions}

This study attempted to quantify the problem of post-COVID symptoms to prepare the healthcare providers for the forthcoming burden. Female sex and comorbid disease conditions are found as risk factors for long post-COVID symptoms. As most patients showed declining symptoms over time, counseling the patient regarding illness will set expectations. However, patients with incapacitating symptoms should be monitored closely. Pragmatic rehabilitation and psychiatric care programs need to be developed based on the prospective research findings.

\section{Additional Information \\ Disclosures}

Human subjects: Consent was obtained or waived by all participants in this study. Institutional Review Board of Bangabandhu Sheikh Mujib Medical University issued approval BSMMU/2020/6317. Animal subjects: All authors have confirmed that this study did not involve animal subjects or tissue. Conflicts of interest: In compliance with the ICMJE uniform disclosure form, all authors declare the following: Payment/services info: The study received a grant from Bangabandhu Sheikh Mujib Medical University with the grant number bsmmu/2020/7231(02). Financial relationships: All authors have declared that they have no financial relationships at present or within the previous three years with any organizations that might have an interest in the submitted work. Other relationships: All authors have declared that there are no other relationships or activities that could appear to have influenced the submitted work.

\section{References}

1. COVID-19 coronavirus pandemic. (2021). Accessed: April 4, 2021: https://www.worldometers.info/coronavirus/.

2. Davido B, Seang S, Tubiana R, de Truchis P: Post-COVID-19 chronic symptoms: a postinfectious entity? Clin Microbiol Infect. 2020, 26:1448-1449. 10.1016\%2Fj.cmi.2020.07.028

3. Sudre CH, Murray B, Varsavsky T, et al.: Attributes and predictors of long-COVID: analysis of COVID cases and their symptoms collected by the covid symptoms study app. medRxiv. 2020, 10.1101/2020.10.19.20214494

4. The Lancet: Facing up to long COVID. Lancet. 2020, 396:1861. 10.1016/S0140-6736(20)32662-3

5. Clinical spectrum of SARS-CoV-2 infection. (2020). Accessed: January 10, 2021: https://www.covid19treatmentguidelines.nih.gov/overview/clinical-spectrum/.

6. Post COVID/long COVID. (2021). Accessed: January 19, 2021: https://www.idsociety.org/covid-19-real-timelearning-network/disease-manifestations--complications/post-covid-syndrome/.

7. Tenforde MW, Kim SS, Lindsell CJ, et al.: Symptom duration and risk factors for delayed return to usual health among outpatients with COVID-19 in a multistate health care systems network - United States, March-June 2020. MMWR Morb Mortal Wkly Rep. 2020, 69:993-998. 10.15585/mmwr.mm6930e1

8. Carvalho-Schneider C, Laurent E, Lemaignen A, et al.: Follow-up of adults with noncritical COVID-19 two months after symptom onset. Clin Microbiol Infect. 2021, 27:258-263. 10.1016/j.cmi.2020.09.052

9. Huang C, Huang L, Wang Y, et al.: 6-month consequences of COVID-19 in patients discharged from 


\section{Cureus}

hospital: a cohort study. Lancet. 2021, 397:220-232. 10.1016/S0140-6736(20)32656-8

10. Lwanga SK, Lemeshow S: Sample Size Determination in Health Studies: A Practical Manual . World Health Organization, Geneva; 1991.

11. Carfì A, Bernabei R, Landi F: Persistent symptoms in patients after acute COVID-19. JAMA. 2020, 324:603605. 10.1001/jama.2020.12603

12. Yelin D, Wirtheim E, Vetter P, et al.: Long-term consequences of COVID-19: research needs. Lancet Infect Dis. 2020, 20:1115-1117. 10.1016/S1473-3099(20)30701-5

13. Ahmed H, Patel K, Greenwood DC, et al.: Long-term clinical outcomes in survivors of severe acute respiratory syndrome and Middle East respiratory syndrome coronavirus outbreaks after hospitalisation or ICU admission: a systematic review and meta-analysis. J Rehabil Med. 2020, 52:jrm00063. 10.2340/16501977-2694

14. Wu X, Dong D, Ma D: Thin-section computed tomography manifestations during convalescence and longterm follow-up of patients with severe acute respiratory syndrome (SARS). Med Sci Monit. 2016, 22:27932799. 10.12659\%2FMSM.896985

15. Lam MH, Wing YK, Yu MW, et al.: Mental morbidities and chronic fatigue in severe acute respiratory syndrome survivors: long-term follow-up. Arch Intern Med. 2009, 169:2142-2147.

10.1001/archinternmed.2009.384

16. Garrigues E, Janvier P, Kherabi Y, et al.: Post-discharge persistent symptoms and health-related quality of life after hospitalization for COVID-19. J Infect. 2020, 81:4-6. 10.1016/j.jinf.2020.08.029

17. Nabavi N: Long covid: how to define it and how to manage it . BMJ. 2020, 370:m3489. Accessed: January 5, 2021: 10.1136/bmj.m3489 